\section{ER calcium stabilizers in NS}

A growing body of literature indicates that calcium dysregulation is a feature of podocytopathies such as nephrotic syndrome (NS); however, little is known about the role of endoplasmic reticulum (ER) calcium efflux on podocyte health. Using a mouse model of ER stress-induced NS, researchers now show that phosphorylation of the type 2 ryanodine receptor (RYR2) in podocytes leads to calcium leak from the ER into the cytosol, and that ER calcium stabilizers can rescue injurious podocyte responses. "We demonstrate that the ER calcium release channel RYR2 undergoes phosphorylation at Ser2808 during ER stress," explains Ying Maggie Chen. "Most importantly, we identify a chemical compound and a novel biotherapeutic protein that can fix leaky RYR2 and inhibit podocyte injury."

To assess the consequences of ER stress on calcium homeostasis in podocytes, Chen and colleagues used a mouse model of NS caused by podocyte-specific expression of a Cys321Arg mutation in LAMB2, which encodes the glomerular basement membrane component laminin $\beta 2$. Primary podocytes from the mutant mice showed upregulation of proteins associated with ER stress and calcium-mediated apoptosis. Further analyses showed that mutant podocytes had increased RYR2 phosphorylation and ER calcium release. "The accelerated podocyte ER calcium efflux due to leaky RYR2 activates cytosolic protease calpain 2, leading to apoptosis, cytoskeleton disruption and podocyte injury," says Chen.

Assessment of the ER calcium release inhibitor, K201, showed that it inhibited RYR2 hyperphosporylation, attenuated the ER calcium leak and reduced apoptosis in podocytes from mutant mice. In vivo, K201 reduced albuminuria, improved indices of kidney function and prevented the development of podocyte pathology in mutant mice. Similarly, administration of recombinant mesencephalic astrocyte-derived neurotrophic factor (MANF) attenuated apoptosis of mutant podocytes. "Our findings suggest that podocyte ER calcium channel stabilizers are an emerging therapeutic strategy to treat NS caused by ER dysfunction," says Chen. "We plan to move forward with this new class of drugs, and will continue efforts to identify additional ER calcium stabilizers to treat podocytopathies."

Susan J. Allison

ORIGINAL ARTICLE Park, S. J. et al. Discovery of endoplasmic reticulum calcium stabilizers to rescue ER-stressed podocytes in nephrotic syndrome. Proc. Natl Acad. Sci. USA https://doi.org/ 10.1073/pnas.1813580116 (2019)

\title{
Gene signatures reveal kidney immune cells
}

Single-cell RNA sequencing (scRNA-seq) is increasingly being used to define cellular phenotypes based on signatures of gene expression. Using this technique, Nir Hacohen, Betty Diamond and colleagues mapped immune cell subsets in the kidneys of patients with lupus nephritis.

The researchers applied scRNA-seq to cells isolated from kidney biopsy samples collected from 24 patients with lupus nephritis and 10 control samples from living donors. The gene expression data defined kidney cell clusters, including B cells, T cells, natural killer (NK) cells, myeloid cells and epithelial cells. CXCR4 was expressed in the majority of infiltrating kidney cells, whereas CX3CR1 was expressed in most myeloid and NK cells.

B cells from lupus nephritis samples ranged from naive to activated cells, but were rare in control kidneys. In addition, the expression of cytotoxic molecules and specific lineage markers identified clusters of NK cells and $\mathrm{CD}^{+} \mathrm{T}$ cells. Exhaustion markers were expressed at higher levels in blood $\mathrm{CD}^{+} \mathrm{T}$ cells than in kidney $\mathrm{CD}^{+}$ $\mathrm{T}$ cells. $\mathrm{CD} 4^{+}$memory $\mathrm{T}$ cells were present in both patients and controls, but patient samples had a larger central memory $\mathrm{CD}^{+}$ $\mathrm{T}$ cell population and higher expression of interferon-stimulated genes (ISGs).

The main myeloid cell cluster in control samples likely represented resident kidney cells and its unique gene expression pattern differed from that of peripheral cell subsets. In patient samples, this myeloid cell cluster expressed higher levels of ISGs and anti-inflammatory genes than in controls. Other myeloid cells identified in patient samples included a cluster of inflammatory cells most similar to CD16 ${ }^{+}$blood monocytes, and cells that either expressed markers of alternative activation or of the dendritic cell lineage.

The gene expression profile of immune cells isolated from the urine of patients with lupus nephritis revealed a high correlation with the gene signature of kidney immune cells. Isolating immune cells from urine might thus serve as a noninvasive method for the analysis of kidney immune cells.

Monica Wang

ORIGINAL ARTICLE Arazi, A. et al. The immune cell landscape in kidneys of patients with lupus nephritis. Nat. Immunol. 20, 902-914 (2019)

\section{IMAGING}

\section{A single-cell, 2D atlas of the normal human kidney using imaging mass cytometry}

Greater understanding of the gene expression and spatial organization of cells within the kidney is expected to aid understanding of normal and disease processes. However, the number of analyses that can be performed is restricted by the limited amount of biopsy tissue. In new research, Nikhil Singh and Lloyd Cantley overcome this limitation by using an imaging mass cytometry (IMC)-based approach to phenotype and quantify diverse cell types within biopsy samples. "Our aim was to develop a reference data set against which quantitative interrogation of diseased tissue could be compared," says Singh.

IMC couples laser ablation of formalinfixed paraffin-embedded tissue to timeof-flight mass spectrometry to generate high-resolution reconstructions of multiple markers on a single section of tissue.

To define cell types within kidneys, the researchers assembled and validated a panel of 23 metal-conjugated markers, which were applied to kidney tissue samples that were considered histopathologically normal. They also developed a machine learning-based technique, termed Kidney-MAPPS, to quantify the cells. "Unsurprisingly, we found that proximal tubular cells are the most abundant cell type in the cortex, and that immune cells vary in their abundance from cortex to medulla," says Singh. "We also identified rare and unexpected cell populations, including a subset of proximal tubule cells that express vimentin and may represent regenerating cells, as well as vascular cells that express WT1 and nestin."

The researchers plan to use this methodology to test hypotheses about the mechanisms of cell death and regeneration and role of immune cells. "IMC coupled to Kidney-MAPPS can provide an immense amount of quantitative data from a single slide; we believe this methodology may unlock a wealth of previously inaccessible information contained in kidney biopsy repositories worldwide," notes Singh.

Susan J. Allison

ORIGINAL ARTICLE Singh, N. et al. Development of a 2-dimensional atlas of the human kidney with imaging mass cytometry. JCl Insight 4, 129477 (2019) 